\title{
Steady Magnetohydrodynamic Equations for Quantum Plasmas
}

\author{
Muhammad Asif, Umar Bashir \\ Department of Physics, COMSATS Institute of Information Technology, Lahore, Pakistan \\ Email: dr.muha.asif@gmail.com, umarbashir85@gmail.com
}

Received September 30, 2012; revised October 30, 2012; accepted November 13, 2012

\begin{abstract}
Steady Magnetohydrodynamic (MHD) Equations of force, density and energy for quantum plasmas have been derived. These equations constitute our Steady Magnetohydrodynamic model for quantum plasmas. All the quantum effects are contained in the last term of quantum force equation and in the last three terms of quantum Energy Equation, so-called Bohm potential and may be valuable for the description of quantum phenomena like tunneling.
\end{abstract}

Keywords: Steady Magnetohydrodynamic; Quantum Plasmas; Inhomogeneous Magnetic Field

\section{Introduction}

In classical plasma physics, fluid models are ubiquitous, with their application ranging from astrophysics to controlled nuclear fusion [1-3]. Magnetohydrodynamics, for instance, provides one of the most useful fluid models, focusing on the global properties of the plasma. The purpose of work [4] is to produce a quantum counterpart of magnetohydrodynamics, starting with the quantum hydrodynamic model for charged particle systems. This may provide yet another approach to the study of the ways in which quantum physics can modify classical plasma physics. However, it must be noted that the quantum hydrodynamic model for charged particle systems was built for non-magnetized systems only [4]. In order to arrive at a quantum modified magnetohydrodynamics, work [4] suggests what the appropriate extension would be of the quantum hydrodynamic model in cases of nonzero magnetic field.

In this paper, we present the Steady Magnetohydrodynamic (MHD) Equations of force, density and energy for quantum plasmas. These equations constitute our Steady Magnetohydrodynamic model for quantum plasmas. All the quantum effects are contained in the last term of quantum force equation and in the last three terms of quantum Energy Equation, so-called Bohm potential and may be valuable for the description of quantum phenomena like tunneling.

\section{Steady Magnetohydrodynamic Equations}

The Steady Magnetohydrodynamic (MHD) equations [5] describe the plasma as a conducting fluid with conduc- tivity which experiences electric and magnetic forces. The fluid is specified by a mass density $\rho$, a flow velocity $V$, and a pressure $P$, which are all functions of space and time. Here, we shall simply draw on the ordinary equations of hydrodynamics. The equation of motion has an additional force per unit volume $J$ due to the net magnetic force on the plasma particles. On fluid scales $\left(\gg \lambda_{D}\right)$ the plasma is overall charge neutral, so that the net electric force is neglibly small. The MHD equations represent the conservation of momentum, the conservation of mass and some equation of state (i.e., an energy relationship). The simplified Steady Magnetohydrodynamic Equations [5] are

$$
\begin{aligned}
& \rho[V \cdot \nabla] V=-\nabla P+J \times B \\
& \nabla \cdot(\rho V)=0 \\
& \nabla \cdot(P V)=0
\end{aligned}
$$

There are a number of assumptions behind the above equations: the energy equation is the adiabatic equation of state with $\gamma$ being the ratio of specific heats $\left(\gamma=\frac{5}{3}\right.$ for a monatomic gas); there are other possible choices for an energy equation such as isothermal $(\gamma=1)$, etc. Ohmic heating J.E has been ignored, i.e., assumed to be less important than the plasma's thermal energy; radiative or other heat losses are also neglected. We have also assumed that the pressure is isotropic which is not generally true when the magnetic field can impose different kinds of motion on the particles parallel and perpendicular to the field direction. Any possible heat flux has been ignored, and there is no relative flow between the differ- 
ent species in the plasma, so that the one fluid description is valid. While the conductivity is usually large due to the mobile electrons, the absence of collisions has the opposite impact on other transport processes such as viscosity, which we have also therefore neglected.

\section{Steady Quantum Force Balance Equation}

The Steady force balance equation for quantum plasma is obtained by putting the pressure $P$ given by $[4,6]$ as

$$
P=p^{k}+p^{o}+p^{Q}
$$

Since the kinetic and osmotic pressures measures the kinetic and osmotic velocity dispersions, we can write from $[4,6]$

$$
p^{k}+p^{o}=p(n)
$$

Equation (5) depend only on the density, we obtain from $[4,6]$

$$
P=p(n)-\frac{\hbar^{2} \rho}{2 m_{i} m_{e}}\left(\frac{\nabla^{2} \sqrt{\rho}}{\sqrt{\rho}}\right)
$$

Using Equations (5) and (6), the Steady force balance Equation (1) for quantum plasma is obtained as

$$
\rho[V \cdot \nabla] V=-\nabla p+J \times B+\frac{\hbar^{2} \rho}{2 m_{i} m_{e}} \nabla\left(\frac{\nabla^{2} \sqrt{\rho}}{\sqrt{\rho}}\right)
$$

The derived Steady quantum force Equation (7) constitute our quantum hydrodynamic model for magnetized systems. All the quantum effects are contained in the last term of Equation (7), the so-called Bohm potential and may be valuable for the description of quantum phenomena like tunneling.

\section{Steady Quantum Density Equation}

The density Equation (2), for quantum plasmas is

$$
(V \cdot \nabla) \rho+\rho \nabla \cdot V=0
$$

This is Steady density equation, which does not contained the quantum effects.

\section{Steady Quantum Energy Equation}

Using Equations (5) and (6), the Steady energy Equation (3), for quantum plasmas is obtained as

$$
[V \cdot \nabla] p
$$$$
+\left(p-\frac{\hbar^{2} \rho}{2 m_{i} m_{e}}\left(\frac{\nabla^{2} \sqrt{\rho}}{\sqrt{\rho}}\right)\right) \nabla \cdot V-\frac{V \hbar^{2} \rho}{2 m_{i} m_{e}} \nabla\left(\frac{\nabla^{2} \sqrt{\rho}}{\sqrt{\rho}}\right)=0
$$

The derived Steady Energy Equation (9) constitute our quantum hydrodynamic model for magnetized systems. All the quantum effects are contained in the last three terms of Equation (9), the so-called Bohm potential and may be valuable for the description of quantum phenomena like tunneling.

In comparison with standard fluid models for charged particle systems, the Bohm potential is the only quantum contribution. In order to have a deeper understanding of the importance of quantum effects, we compare Equations (7)-(9) with the rescaling of ideal quantum magnetohydrodynamic Equation (46) of [4], and obtained a nondimensional parameter $H=\frac{\hbar \omega_{c}}{\sqrt{m^{2}} V_{A}^{2}}$, measuring the relevance of quantum effects. Where $V_{A}$ is a Alfvén velocity and $\omega_{c}$ is the cyclotron velocity. While for ordinary plasmas $H$ is negligible, for dense astrophysical plasmas, $H$ can be of the order unity or more. Hence, in dense astrophysical plasmas [7] such as the atmosphere of neutron stars or the interior of massive white dwarfs, quantum corrections to magnetohydrodynamics can be experimentally important.

\section{Conclusion}

Steady Magnetohydrodynamic (MHD) Equations of force, density and energy for quantum plasmas have been derived. The Steady force, density and Energy equations constitute our reduced quantum hydrodynamic model for magnetized systems. All the quantum effects are contained in the last term of force Equation (7) and in the last two terms of Energy Equation (9), so-called Bohm potential and may be valuable for the description of quantum phenomena like tunneling.

\section{REFERENCES}

[1] D. R. Nicholson, “Introduction to Plasma Theory,” Wiley, New York, 1983.

[2] J. A. Bittencourt, "Fundamentals of Plasma Physics,” National Institute for Space Research, São José dos Campos, 1995.

[3] M. Asif, X. Gao, J. Li, et al., "Plasma Density Behavior with New Graphite Limiters in the Hefei Tokamak-7,” Physics of Plasmas, Vol. 12, No. 8, 2005, Article ID: 082502. doi:10.1063/1.1995627

[4] F. Haas, "A Magnetohydrodynamic Model for Quantum Plasmas,” Physics of Plasmas, Vol. 12, No. 6, 2005, Article ID: 062117.

[5] S. J. Schwartz, C. J. Owen and D. Burgess, "Astrophysical Plasmas Astronomy Unit, Queen Mary,” University of London, London, 2004, p. 35.

[6] F. Haas, “An Introduction to Quantum Plasmas,” Brazilian Journal of Physics, Vol. 41, No. 4, 2011, p. 349. doi:10.1007/s13538-011-0043-0

[7] G. Chabrier, F. Douchin and A. Y. Potekhin, "Dense Astrophysical Plasmas,” Journal of Physics: Condensed Matter, Vol. 14, No. 40, 2002, p. 9133. doi:10.1088/0953-8984/14/40/307 